\title{
On an inverse boundary value problem*
}

\author{
ALBERTO P. CALDERÓN
}

Department of Mathematics, The University of Chicago

\begin{abstract}
This paper is a reprint of the original work by A. P. Calderón published by the Brazilian Mathematical Society (SBM) in ATAS of SBM (Rio de Janeiro), pp. 65-73, 1980. The original paper had no abstract, so this reprint to be truthful to the original work is published with no abstract.
\end{abstract}

Mathematical subject classification: 35J25, 35Q60, 47F05, 86A20, 86A22.

Key words: inverse problems, boundary value problems, identification problem, elliptic equations.

In this note we shall discuss the following problem. Let $D$ be a bounded domain in $\mathbb{R}^{n}, n \geq 2$, with Lipschitzian boundary $d D$, and $\gamma$ be a real bounded measurable function in $D$ with a positive lower bound. Consider the differential operator

$$
L_{\gamma}(w)=\nabla \cdot(\gamma \nabla w)
$$

acting on function of $H^{1}(D)$ and the quadratic form $Q_{\gamma}(\phi)$, where the functions in $H^{1}\left(\mathbb{R}^{n}\right)$, defined by

$$
\begin{gathered}
Q_{\gamma}(\phi)=\int_{D} \gamma(\nabla w)^{2} d x, \quad w \in H^{1}\left(\mathbb{R}^{n}\right),\left.w\right|_{d D}=0 \\
L_{\gamma} w=\nabla \cdot(\gamma \nabla w)=0 \text { in } D .
\end{gathered}
$$

\#693/06. Received: 14/XI/06.

*We thank the Brazilian Mathematical Society (SBM) for the permission to reproduce this article originally published at the "Seminar on Numerical Analysis and its Application to Continuum Physics" in the ATAS of SBM (Rio de Janeiro), 1980, pp. 65-73. 
The problem is then to decide whether $\gamma$ is uniquely determined by $Q_{\gamma}$ and to calculate $\gamma$ in terms $Q_{\gamma}$, if $\gamma$ is indeed determined by $Q_{\gamma}$.

This problem originates in the following problem of electrical prospection. If $D$ represents an inhomogeneous conducting body with electrical conductivity $\gamma$, determine $\gamma$ by means of direct current steady state electrical measurements carried out on the surface of $D$, that is without penetrating $D$. In this physical situation $Q_{\gamma}(\phi)$ represents the power necessary to maintain an electrical potential $\gamma$ in $\partial D$.

In principle $Q_{\gamma}$ can be determined through measurements effected on $d D$ and contains all information about $\gamma$ which can be thus obtained.

But let us return to our mathematical problem. Let us introduce the following norms in the space of functions $\gamma$ on $d D$ and in the space of quadratic forms $Q(\phi)$

$$
\begin{aligned}
\|\phi\|^{2} & =\int_{D}|\nabla u|^{2} d x ;\left.u\right|_{d D}=\phi, \Delta u=0 \text { in } D . \\
\|Q\| & =\sup _{\|\phi\| \leq 1}|Q(\phi)|
\end{aligned}
$$

Then the mapping

$$
\Phi: \gamma \rightarrow Q_{\gamma}
$$

is bounded and analytic in the subset of $L^{\infty}(D)$ consisting of functions which are real and have a positive lower bound. Our goal is then to determine whether $\Phi$ is injective, and invert $\Phi$ if this is the case. This we are yet unable to do and is, as far as we know, an open problem. However we shall show that $\left.d \Phi\right|_{\gamma=\text { const }}$ is indeed injective, that is, the linearized problem has an affirmative answer. If $\left.d \Phi\right|_{\gamma=\text { const }}$, which is a linear operator, had a closer range, one could conclude that $\Phi$ itself is injective in a sufficiently small neighborhood of $\gamma=$ const. But the range of $\mathrm{d} \Phi$ is not closed and the desired conclusion cannot be obtained in this fashion. Nevertheless, as we shall see below, if $\gamma$ is sufficiently close to a constant, it is nearly determined by $Q_{\gamma}$ and in some cases it can be calculated with an error much smaller than $\| \gamma-$ const $\|_{L^{\infty}}$.

To show this let us first obtain an expression for the solution of the equation

$$
L_{\gamma}(W)=\nabla \cdot(\gamma \nabla W),\left.W\right|_{d D}=\phi \cdot \gamma=1+\delta
$$


Let $W=u+v$, where $\Delta u=L_{1} u=0,\left.u\right|_{d D}=\phi$. Then

$$
L_{\gamma}(W)=L_{1+\gamma}(u+v)=L_{1} v+L_{\gamma} v+L_{\gamma} u=0
$$

Since $\left.u\right|_{d D}=W \mid d D$ we have $\left.v\right|_{d D}=0$ and $v \in H_{0}^{1}(D)$, the closure in $H^{1}\left(\mathbb{R}^{n}\right)$ of functions of $C^{\infty}$ with support in $D$. But $L_{1}$, as an operator from $H_{0}^{1}(D)$ into $H^{-1}(D)$, has a bounded inverse $G$, and from the last expression we obtain

$$
v+G L_{\delta} v=-G L_{\delta} u
$$

and

$$
v=-\left[\sum_{0}^{\infty}(-1)^{j}\left(G L_{\delta}\right)^{j}\right]\left(G L_{\delta} u\right)
$$

Since for $W \in H_{0}^{1}(D),\left\|L_{\delta} W\right\|_{H^{-1}(D)} \leq\|\delta\|_{L^{\infty}}\|W\|_{H_{0}^{1}(D)}$ if $A$ denotes the norm of $G$, the series above will converge for $\|\delta\|_{L^{\infty}} A<1$ and

$$
\|v\|_{H^{-1}(D)} \leq \frac{A\|\delta\|_{L^{\infty}}\|\phi\|}{1-A\|\delta\|_{L^{\infty}}}
$$

From (1) it follows that $\phi$ is analytic at $\gamma=1$. The same argument would show that $\phi$ is analytic at any other point $\gamma$.

Next let us calculate $\left.d \phi\right|_{\gamma=1}$. We have

$$
\begin{aligned}
Q_{1+\delta}(\phi)= & \int_{D}(1+\delta)|\nabla W|^{2} d x=\int_{D}\left[(1+\delta)|\nabla u|^{2}+2(\nabla u \cdot \nabla u)\right. \\
& \left.+2 \delta(\nabla u \cdot \nabla v)+(1+\delta)|\nabla v|^{2}\right] d x
\end{aligned}
$$

The contribution of the second term in the integrand of the last integral vanishes on account of the fact that $\Delta u=0$. Furthermore, from (1) one sees readily that the parts linear in $\delta$ of the last two terms in the integrand vanish. Thus setting $d \gamma=\delta$ we obtain

$$
\left.d Q_{\gamma}(\phi)\right|_{\gamma=1}=\int_{D} \delta|\nabla u|^{2} d x, \quad \Delta u=0,\left.\quad u\right|_{d D}=\phi
$$

To show that $\left.d Q_{\gamma}(\phi)\right|_{\gamma=1}$ is injective, we merely have to show that if the last integral vanishes for all $u$ with $\Delta u=0$ then $\delta=0$. But if the integral vanishes for all such $u$, then we also have

$$
\int_{D} \delta\left(\nabla u_{1} \cdot \nabla u_{2}\right) d x=0
$$


whenever $\Delta u_{1}=\Delta u_{2}=0$ in $D$. Now let $Z$ be any vector in $\mathbb{R}^{n}$ and $\underline{a}$ another vector such that $|\underline{a}|=|Z|,(\underline{a} . Z)=0$. Then the functions

$$
u_{1}(x)=e^{\pi i(Z \cdot x)+\pi(\underline{a} . x)}, \quad u_{2}=e^{i(Z \cdot x)-(\underline{a} . x)} .
$$

are harmonic, and substituting in (3) we obtain

$$
2 \pi|Z|^{2} \int_{D} \delta(x) e^{2 \pi i(Z . x)} d x=0, \quad \forall Z
$$

whence it follows that $\delta=0$.

Now let us return to $Q_{\gamma}(W)$. We set again $\gamma=1+\delta$ and introduce the bilinear form

$$
B\left(\phi_{1}, \phi_{2}\right)=\frac{1}{2}\left[Q_{\gamma}\left(W_{1}+W_{2}\right)-Q_{\gamma}\left(W_{1}\right)-Q_{\gamma}\left(W_{2}\right)\right]
$$

and setting $W_{j}=u_{j}+v_{j}, j=1,2, \Delta u_{j}=0,\left.u_{j}\right|_{d D}=\phi_{j}$ we obtain

$$
\begin{aligned}
B\left(\phi_{1}, \phi_{2}\right)= & \int_{D}(1+\delta)\left(\nabla u_{1} . \nabla u_{2}\right)+\delta\left[\left(\nabla u_{1} . \nabla v_{2}\right)+\left(\nabla u_{2} . \nabla v_{1}\right)\right] \\
& +(1+\delta)\left(\nabla v_{1} . \nabla v_{2}\right) d x
\end{aligned}
$$

Now, substitution of the exponentials in (5) for $u_{1}$ and $u_{2}$ in the preceding expression (taking $\underline{a}$ to be a function of $Z$ such that $|a|=|Z|,(\underline{a} . Z)=0$ ) yields

$$
\hat{\gamma}(Z)=\hat{F}(Z)+R(Z)
$$

where $\hat{\gamma}(Z)$ is a Fourier transform of $\gamma$ extended to be zero outside $D$, the function

$$
\hat{F}(Z)=\frac{1}{2 \pi^{2}|Z|^{2}} B\left(e^{i \pi(Z . x)+\pi(a . x)}, e^{i \pi(Z . x)-\pi(a . x)}\right)
$$

is known and, as follows readily from (2),

$$
|R(Z)| \leq C\|\delta\|_{L^{\infty}}^{2} \mathrm{e}^{2 \pi r|Z|}
$$

provided that $A\|\delta\|_{L^{\infty}} \leq 1-\varepsilon$, where $C$ depends only on $D$ and $\varepsilon$, and $r$ is the radius of the smallest sphere containing $D$. Now $R(Z)$ is too large to permit estimating $\gamma(x)$. However, under favorable circumstances it is still possible to obtain satisfactory information about $\gamma$. Choose $\alpha, 1<\alpha<2$, then for

$$
|Z| \leq \frac{2-\alpha}{2 \pi r} \log \frac{1}{\|\delta\|_{L^{\infty}}}=\sigma
$$


we have $|R(Z)| \leq C\|\delta\|_{L^{\infty}}^{\alpha}$. Let $\eta$ be a function such that $\hat{\eta} \in C^{\infty}$, supp $\hat{\eta} \subset$ $\{|Z| \leq 1\} \hat{\eta}(0)=1$, and let $\eta_{\sigma}=\sigma^{n} \eta(\sigma Z)$. Then we have

$$
\hat{\gamma}(Z) \hat{\eta}\left(\frac{Z}{\sigma}\right)=\hat{F}(Z) \hat{\eta}\left(\frac{Z}{\sigma}\right)+R(Z) \hat{\eta}\left(\frac{Z}{\sigma}\right)
$$

and

$$
\left(\gamma * \eta_{\sigma}\right)(x)=\left(F * \eta_{\sigma}\right)(x)+\rho(x)
$$

where $*$ denotes convolution and

$$
|\rho(Z)| \leq C\|\delta\|_{L^{\infty}}^{\alpha} \int\left|\hat{\eta}\left(\frac{Z}{\sigma}\right)\right| d Z=C_{1}\|\delta\|_{L^{\infty}}^{\alpha}\left[\log \frac{1}{\|\delta\|_{L^{\infty}}}\right]^{n}
$$

where $C_{1}$ depends only on $D, \alpha$ e $\varepsilon$.

Thus if $\|\delta\|_{L^{\infty}}$ is sufficiently small, (9) gives an approximation for $\gamma * \eta_{\sigma}$ with an error which is much smaller than $\|\delta\|_{L^{\infty}}$. Clearly, if $\|\delta\|_{L^{\infty}}$ is small then $\sigma$ is large and $\gamma * \eta_{\sigma}$ is itself in some sense, a good approximation to $\gamma$.

Approximations to the function $\gamma$ itself may be obtained if one assumes that $\gamma$, extended to be equal to 1 outside $D$, is in $C^{m}, m>n$. In this case one obtains

$$
\hat{\delta}(Z)=\hat{F}_{1}(Z)+\hat{R}(Z)
$$

where $F_{1}$ is known and $R(Z)$ is the same as in (6). One then calculates $\delta(x)$ by integrating over $|Z| \leq \sigma$ with $\sigma$ as in (8) and estimates the error by using the decay of $\hat{\delta}$ at $\infty$. Thus one obtains

$$
\gamma(x)=F_{2}(x)+\sigma(x)
$$

where $F_{2}(x)$ is known and

$$
|\rho(x)| \leq C\|\delta\|_{L^{\infty}}^{\alpha}\left[\log \frac{1}{\|\delta\|_{L^{\infty}}}\right]^{n}+C M\left[\log \frac{1}{\|\delta\|_{L^{\infty}}}\right]^{m+n}
$$

where $M$ is a bound for the derivatives of order $m$ of $\gamma$.

\section{REFERENCES}

We have been unable to find treatments of the problem discussed above in the literature, at least not in the general setting in which we are interested. Similar problems have been studied in the papers listed below. 
[1] J.R. Cannon, Determination of certain parameters in heat conduction problems. J. Math. Anal. Appl., 8 (1964), 188-201.

[2] J.R. Cannon, Determination of an unknown coefficient in a parabolic differential equation. Duke Math. J., 30 (1963), 313-323.

[3] J.R. Cannon, Determination of the unknown coefficient $k(u)$ in the equation $V \cdot k(u) \nabla u=0$ from overspecified boundary data. J. Math. Anal. Appl., 18 (1967), 112-114.

[4] J.R.Cannon and Paul Du Chateau, Determining unknown coefficients in a non-linear heat conduction problem. SIAM J. Appl. Math., 24(3) (1973), 298-314.

[5] Jim Douglas, Jr and G.F. Jones, Determination of a coefficient in a parabolic differential equation, Part. II: numerical approximation. J. Math. Mech., 11 (1962), 919-926.

[6] B.F. Jones, Various methods for finding unknown coefficients in parabolic differential equations. Comm. Pure Appl. Math., 16 (1963), 33-44. 